\title{
Etiology, clinical outcome, and laboratory features in children with neutropenia: Analysis of 104 cases
}

\author{
Giulia Angelino ${ }^{1}$, Roberta Caruso ${ }^{2}$, Patrizia D'Argenio ${ }^{1}$, Francesca Ippolita Calò Carducci ${ }^{1}$, \\ Roberto Pascone ${ }^{3}$, Marina Lanciotti ${ }^{4}$, Caterina Cancrini ${ }^{1}$, Paolo Palma ${ }^{1}$, Alessandro Aiuti ${ }^{1}$, \\ Paolo Rossi ${ }^{1} *$ \& Andrea Finocchi ${ }^{1} *$
}

${ }^{1}$ University-Hospital Pediatric Department, Bambino Gesù Children's Hospital IRCCS and University of Rome, 'Tor Vergata' School of Medicine, Rome, Italy; ${ }^{2}$ Department of Pediatric Hematology and Oncology, Bambino Gesù Children's Hospital IRCCS, Rome, Italy; ${ }^{3}$ Department of Pediatrics, University of Rome 'Sapienza' and Policlinico Umberto I, Rome, Italy; ${ }^{4}$ Department of Pediatric Hematology and Oncology, Giannina Gaslini Children's Hospital, Genoa, Italy

To cite this article: Angelino G, Caruso R, D’Argenio P, Calò Carducci FI, Pascone R, Lanciotti M, Cancrini C, Palma P, Aiuti A, Rossi P, Finocchi A. Etiology, clinical outcome, and laboratory features in children with neutropenia: Analysis of 104 cases. Pediatr Allergy Immunol 2014: 25: 283-289.

\section{Keywords}

acute neutropenia; autoimmune neutropenia; children; chronic idiopathic neutropenia; congenital neutropenia; post-infectious neutropenia; severe congenital neutropenia

\section{Correspondence}

Andrea Finocchi, University-Hospital Pediatric Department, Unit of Immunology and Infectious Disease, Bambino Gesù Children's Hospital IRCCS, and University of Rome 'Tor Vergata' School of Medicine, Piazza Sant'Onofrio, 4 - 00165 Rome, Italy Tel.: +3906 68592935

Fax: +3906 68592508

E-mail: andrea.finocchi@uniroma2.it

*These authors share seniorship.

Accepted for publication 5 November 2013

DOI:10.1111/pai.12177

\begin{abstract}
Background: Neutropenia is not uncommon in childhood. The aim of our study was to analyze the underlying causes of neutropenia and to evaluate its clinical significance in a series of children referred to our center.

Methods: One hundred and four consecutive children with neutropenia were enrolled in this study. Clinical and laboratory features were analyzed.

Results: The majority of patients $(63.5 \%)$ showed chronic neutropenia. Among all chronic forms, the most frequent was chronic idiopathic neutropenia (CIN), followed by autoimmune neutropenia (AIN). Congenital neutropenia was identified in 6 patients. Acute neutropenia was mainly due to infections.Overall, at the time of first detection, neutropenia was more frequently severe or moderate. One-third of our patients who presented with severe neutropenia were ultimately diagnosed with a postinfectious acute form. Conversely, nearly half patients with CIN, AIN, or congenital neutropenia showed moderate/mild neutropenia at onset.Among patients with AIN and CIN, nearly half recovered between 7 months and 46 months and approximately one-fourth experienced infectious episodes during follow-up. No significant difference was noticed in terms of mean ANC between patients with and without remission, neither between patients with and without infections.

Conclusions: Our study confirms the great etiological heterogeneity of neutropenia in children. We could not demonstrate a correlation between ANC level at onset and the underlying disorder, nor a correlation between mean ANC and duration of neutropenia or infectious episodes during follow-up. Neutropenia remains a disease of concern to pediatricians, requiring several laboratory investigations, prolonged follow-up, and, in few cases, advanced molecular methods.
\end{abstract}

Neutropenia, defined by an absolute neutrophil count (ANC) $<1 \times 10^{9} / 1$ in infants and $<1.5 \times 10^{9} / 1$ thereafter, is not uncommon in pediatric patients (1). Among black populations, lower cutoff values must be considered, because their circulating neutrophils are physiologically reduced $(2,3)$. Neutropenia can be defined as acute or chronic depending on its duration, lower or higher than 3 or 6 months according to different authors (4-8). The severity of neutropenia depends on the ANC: For patients older than $1 \mathrm{yr}$ of age, neutropenia is defined mild with an ANC between 1 and $1.5 \times 10^{9} / 1$, moderate with an ANC between 0.5 and $1 \times 10^{9} / 1$, and severe when ANC is below $0.5 \times 10^{9} / 1(1)$. It is widely accepted that infective diathesis, in terms of frequency and severity of infections, is proportional to the degree of neutropenia $(1,9)$. Nevertheless, a lot of children with moderate-severe isolated neutropenia have a benign course $(4,6,7,10-14)$, meaning that other factors may influence susceptibility to infection, such as the speed of onset and the duration of neutropenia, the bone marrow myeloid reserves, the absolute circulating monocyte count, and the functional status of phagocytes $(1,9,15)$.

A reduction in circulating neutrophils is caused by several inherited and acquired conditions (16). Noteworthy a large number of neutropenia remains of unknown origin, defined as 'idiopathic'. The differential diagnosis of neutropenia some- 
times requires much time and a lot of investigations. Moreover, even when the cause of neutropenia is identified, clinicians have to handle other problems, such as treatment and follow-up schedule. Those aspects still remain controversial and strongly influenced by the single patient's characteristics as well as the clinician's personal experience. In 1994, the International Registry of Severe Chronic Neutropenia (SCNIR) was born to watch over severe chronic forms (17).

This study was undertaken to analyze the underlying causes and the clinical significance of neutropenia in a series of children referred to the Unit of Immunology and Infectious Disease and Unit of Hematology of a large pediatric hospital. To our knowledge, there are few studies examining globally these features in children with neutropenia; the majority of papers focus on fewer issues or specific etiologic categories $(4,6,7,11,18-22)$.

\section{Methods}

Our population is composed by 104 patients, referred to the Unit of Immunology and Infectious Disease and Unit of Hematology of a large pediatric hospital to investigate about a recently detected neutropenia. Chemotherapy-induced neutropenia and those secondary to lympho-myeloproliferative disorders and myelodysplastic syndromes were excluded. The study is a retrospective non-structured analysis of outpatient and inpatient charts of children diagnosed with neutropenia from 2007 to 2010, excluding patients without sufficient diagnostic or follow-up $(n=16)$. Overall, $53 \%$ of children were diagnosed during hospitalization in our pediatric hospital, while the others were referred as outpatients.

We performed a multistep diagnostic workup, on the basis of the recommendations of Italian Association of Pediatric Hematology and Oncology (Associazione Italiana EmatoOncologia Pediatrica, AIEOP) for diagnosis and follow-up of neutropenia in children, which have been recently published by Fioredda et al. (16). Data collection included as follows:

1 Family history, ethnic origin, occurrence of other neutropenia cases, consanguinity.

2 Personal history focused on occurrence of viral and bacterial infections (onset, number, type, site, frequency and severity and cyclic pattern of infections) as well as on drug assumption (type, duration, dose), particularly those indicated as to be associated with neutropenia.

3 Clinical examination, including anthropometry, performed at entry and during follow-up. In particular, clinical findings suggestive for congenital neutropenia were carefully searched for (dysmorphic features, psychomotor development, skeletal abnormalities, albinism, warts, hearth function, liver and spleen size, presence of enlarged lymph nodes, neurological symptoms).

4 Laboratory examinations: ANC monitoring and first-level investigations, including liver and renal function, serum electrolytes, C-reactive protein, fasting glucose, immunoglobulins level, antineutrophil antibodies with indirect granulocyte immunofluorescence test (GIFT), antinuclear antibodies, serology and/or DNA/RNA analysis of some viral pathogens (CMV, EBV, HHV6, parvovirus B19, HIV) and hemogasanalysis.
5 Bone marrow evaluation (morphologic, virologic analysis, cytogenetic, and immunophenotype) was performed in case of confirmed neutropenia with non-informative first-level investigations and no signs or symptoms specific for an associated disease.

6 If the patients showed signs and symptoms and/or biochemical signs suggestive for an associated disease, advanced-level investigations were performed according to possible etiology (16). For example in case of suspected immunodeficiency, standard peripheral blood flow cytometry analysis $(\mathrm{CD} 3+$, $\mathrm{CD} 4+, \mathrm{CD} 8+, \mathrm{CD} 19+, \mathrm{CD} 16+56+$ ) was performed, as well as $\mathrm{IgG}$ subclasses and evaluation of specific antibody responses toward vaccination; when indicated, we also performed CD40 ligand expression, determination of naïve CD45RA and memory subsets on CD4 + and CD8 + T cells, peripheral B-cell subpopulation phenotyping, and molecular analysis. Advanced metabolic examination included urinary organic acids, urinary and serum amino acids, and other supplementary investigations addressed by clinical consultants of metabolic disease, to exclude the metabolic diseases usually associated with neutropenia (glycogenosis Ib, organic acidosis, tyrosinemia, Barth syndrome, Gaucher disease and aminoacidopathies). The following microbiology investigations were performed on body fluid cultures, according to clinical indications: blood culture and/or rapid molecular PCR assays for bacterial and fungal DNA, urine culture, pharyngeal bacterial swab. When marrow morphology showed maturation arrest at the stage of pro-myelocyte/ myelocyte and/or the presence of a cytogenetic clone or significantly reduced cellularity, ELANE and HAX-1 mutations were tested. Other molecular analysis depended on clinical or laboratory features specific for other genetic forms of neutropenia.

Neutropenia was considered 'acute' if lasting $<6$ months and 'chronic' if lasting more.

For statistical analysis, we used standard parameters such as mean, median, range, s.d., Fisher's exact test, Student's $t$-test, and Kaplan-Meier test.

\section{Results}

We analyzed data from 104 patients. Fifty-seven (54.8\%) were male and $47(45.2 \%)$ female. Most of them were of Italian nationality $(92.3 \%)$, except for 4 African children (2 Libyan, 1 Egyptian, 1 Somali), 2 from East Europe, 1 from Colombia, and 1 from India. The age at onset of neutropenia was notably early, $48 \%$ before the age of $1 \mathrm{yr}, 24 \%$ between 12 and 24 months, $28 \%$ over 24 months (global range from 1 months to $17 \mathrm{yrs}$ ) (Fig. 1). Neutropenia was detected during evaluations for different clinical conditions: acute or recurrent infections were the main reasons $(77 \%)$, while in $23 \%$ of cases, neutropenia was incidental.

Most patients showed a chronic course with persistence of neutropenia over 6 months $(n=66,63.5 \%)$. In these patients, follow-up period ranged between 7 and 84 months (mean 23.7 months, median 15 months), while in acute forms, it was extended until 1 month after resolution. The etiologies of acute and chronic neutropenia in our cohort are shown in Table 1. 
Acute neutropenia $(\mathrm{n}=38)$ was mainly due to infections $(\mathrm{n}=26,68.4 \%)$, while one-third remained of unknown etiology.

Post-infectious neutropenia was diagnosed in patients with clinical and biochemical features of infection who showed a reduction in ANC during their acute illness $(n=28)$. Neutropenia in this group was frequently severe $(50 \%)$ or moderate $(36 \%)$. The responsible pathogen was identified in half of the patients (Table 2). Among them, almost all $(n=13)$ had viral infection, mainly from herpes viruses spp, with positive titers of specific IgM antibodies or positive PCR on blood/serum, and one patient had bacterial infection due to Neisseria meningitidis. In half of patients, no infectious agent was identified, although a bacterial origin was suggested on the basis of clinical and laboratory findings. Only in two patients, both infected by parvovirus B19, neutropenia lasted more than 6 months, and in one of them, it resolved after IVIg treatment.

Three patients experienced drug-induced neutropenia after treatment with ganciclovir for CMV congenital infection. One of them, who accidentally received drug overdose, showed chronic neutropenia lasting 8 months.

Among all chronic forms, the most frequent was chronic idiopathic neutropenia (CIN) $(\mathrm{n}=33,50 \%)$, followed by autoimmune neutropenia (AIN) $(\mathrm{n}=22,33.3 \%)$. The diagnosis of AIN was established after finding positive antineutrophil antibodies, while CIN was diagnosed by exclusion, after normal first- and second-level investigations. Neither physical

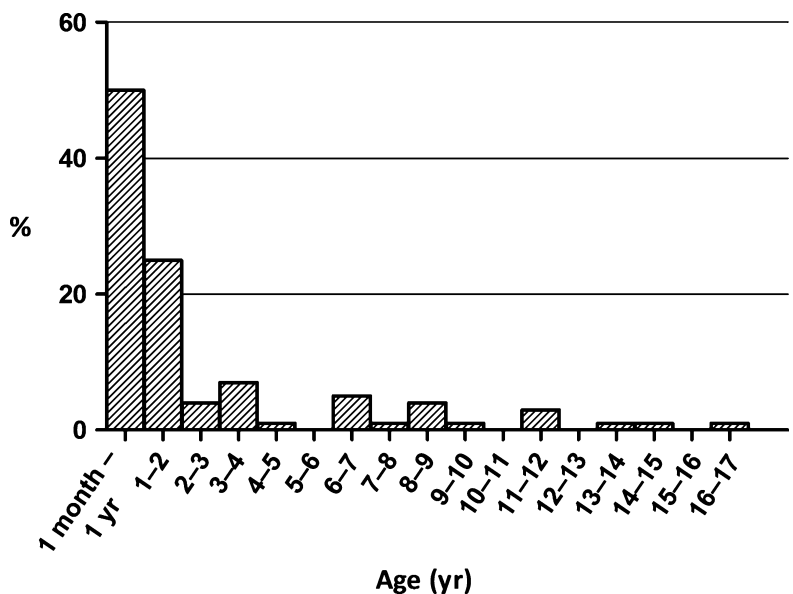

Figure 1 Age distribution at onset of neutropenia. abnormalities (i.e., dysmorphic features, albinism, heart or skeletal anomalies, uropathy, etc.) nor signs/symptoms or laboratory data of a specific disorder were detected, thus

Table 2 Characteristics of Post-infectious neutropenia

\begin{tabular}{|c|c|c|c|c|}
\hline $\mathrm{Pt}$ & Infection & $\begin{array}{l}\text { Age at } \\
\text { diagnosis } \\
\text { (months) }\end{array}$ & $\begin{array}{l}\text { ANC at } \\
\text { diagnosis } \\
(/ \mu l)\end{array}$ & $\begin{array}{l}\text { Duration of } \\
\text { neutropenia } \\
\text { (months) }\end{array}$ \\
\hline \multicolumn{5}{|c|}{ Known pathogen } \\
\hline 1 & HHV-6 & 11 & 700 & 0.5 \\
\hline 2 & HHV-6 & 3 & 100 & 0.25 \\
\hline 3 & HHV-6 & 18 & 330 & 2.5 \\
\hline 4 & HHV-6 & 12 & 300 & 5 \\
\hline 5 & HHV-6+ CMV & 5 & 210 & 3 \\
\hline 6 & HHV-6+ EBV & 36 & 1000 & 0.3 \\
\hline 7 & EBV & 9 & 520 & 3 \\
\hline 8 & EBV & 168 & 820 & 2.6 \\
\hline 9 & VZV & 43 & 1200 & 0.5 \\
\hline 10 & VZV & 1.5 & 440 & 3.5 \\
\hline 11 & HSV & 13 & 400 & 0.6 \\
\hline 12 & Neisseria meningitidis & 96 & 1100 & 2 \\
\hline 13 & Parvovirus B19 & 96 & 830 & $>6 *$ \\
\hline 14 & Parvovirus B19 & 72 & 420 & $>6^{*}$ \\
\hline \multicolumn{5}{|c|}{ Unknown pathogen } \\
\hline 1 & $\begin{array}{l}\text { Upper respiratory } \\
\text { infection }\end{array}$ & 12 & 165 & 1 \\
\hline 2 & $\begin{array}{l}\text { Upper respiratory } \\
\text { infection }\end{array}$ & 13 & 490 & 0.1 \\
\hline 3 & $\begin{array}{l}\text { Upper respiratory } \\
\text { infection }\end{array}$ & 8 & 830 & 0.5 \\
\hline 4 & $\begin{array}{l}\text { Upper respiratory } \\
\text { infection }\end{array}$ & 6 & 380 & 6 \\
\hline 5 & $\begin{array}{l}\text { Upper respiratory } \\
\text { infection }\end{array}$ & 108 & 970 & 0.25 \\
\hline 6 & $\begin{array}{l}\text { Upper respiratory } \\
\text { infection }\end{array}$ & 12 & 490 & 0.6 \\
\hline 7 & Isolated fever & 25 & 1420 & 0.5 \\
\hline 8 & Isolated fever & 3 & 520 & 5 \\
\hline 9 & Isolated fever & 12 & 380 & 0.2 \\
\hline 10 & Urinary tract infection & 15 & 840 & 3 \\
\hline 11 & Urinary tract infection & 4 & 210 & 1.5 \\
\hline 12 & Sepsis & 10.5 & 920 & 1.5 \\
\hline 13 & Sepsis & 6 & 190 & 2 \\
\hline 14 & Arthritis & 2 & 890 & 1 \\
\hline
\end{tabular}

*The two patients infected by parvovirus B19 showed chronic neutropenia.

Table 1 Etiologies of acute and chronic neutropenia

\begin{tabular}{lcclcc}
\hline Acute & $\mathrm{N}$ & $\%$ & Chronic & $\mathrm{N}$ \\
\hline Post-infectious neutropenia & 26 & 68.4 & Chronic idiopathic neutropenia & 33 & $\%$ \\
Idiopathic neutropenia & 10 & 26.3 & Autoimmune neutropenia & 22 & 50 \\
Drug-induced neutropenia & 2 & 5.3 & Congenital neutropenia & 33.3 \\
& & & Benign familial neutropenia & 9 \\
& & & Post-infectious neutropenia & 2 & 3 \\
Total & 38 & 100 & Drug-induced neutropenia & 1 & 1.5 \\
\hline
\end{tabular}


excluding forms of neutropenia associated with syndromes or other diseases (i.e., metabolic). Overall, 32 of 55 (58\%) patients with AIN and CIN underwent bone marrow aspiration for morphology and, in some cases, also for cytogenetic, immunophenotyping, and virologic. In AIN, bone marrow resulted normal or rich with granuloblastic hyperplasia, while in CIN, a normal bone marrow morphology predominated. In particular, 20 of 33 CIN patients were tested for bone marrow. The other 13 patients were not tested either because neutropenia eventually resolved $(n=6)$, patient's parents did not agree with aspiration $(n=2)$, patients were lost to follow up $(n=3)$, or the exam was not performed yet at time of the study analyses $(\mathrm{n}=2)$.

Congenital neutropenia was diagnosed in 6 children (Table 3). In 3 patients, severe congenital neutropenia (SCN) was identified by molecular analysis of ELANE gene. The other 3 patients were, respectively, diagnosed with $\mathrm{X}$-linked hyperIgM syndrome (CD40L mutation), methylmalonic aciduria, and suspected Cohen syndrome.

Benign familial neutropenia was diagnosed in two patients, based on their ethnicity, the relatively mild level of neutropenia, and a similar reduction in ANC detected in their parents.

Globally, in the whole cohort, at the time of first detection, neutropenia was more frequently severe or moderate than mild (severe $\mathrm{n}=42,45 \%$; moderate $\mathrm{n}=40,43 \%$; mild $\mathrm{n}=11$, $12 \%$ ). In 11 patients, onset data were not available (onset ANC not specified in patient's history). The etiologies of neutropenia in children with a ANC $<0.5 \times 10^{9} / 1$ at first evaluation are shown in Fig. 2: One-third had post-infectious neutropenia, the others were almost all affected by acquired chronic forms CIN and AIN, and only two patient were diagnosed with congenital neutropenia (one SCN and one methylmalonic aciduria). Indeed, 4 of 6 patients with congenital neutropenia (including two with ELANE mutation) had ANC above $0.5 \times 10^{9} / 1$ on initial evaluations. The acquired chronic forms, CIN and AIN, presented with ANC above $0.5 \times 10^{9} / 1$, respectively, in $60.7 \%$ and $26.3 \%$ of patients (Fig. 3).

Table 4 summarizes demographic and laboratory data of the 55 patients with AIN and CIN. The mean age at onset was 20 months (range 2-156) in AIN and 31 months (range 1-141) in CIN. The ANC at onset of neutropenia was different between the two groups with a mean of $314 \mu \mathrm{l}$ (range 100-700) in AIN vs. $518 \mu \mathrm{l}$ (range 10-1270) in CIN $(\mathrm{p}=0.01)$. The average follow-up period for AIN and CIN patients was 25 and 20 months, respectively. In particular, $63.6 \%$ of AIN patients and $57.6 \%$ of CIN patients were followed up beyond the completed second year of age. Remission of neutropenia was observed in both groups, but AIN patients showed a lower remission rate during a 24-month follow-up $(p=0.027)$ (Fig. 4). Among patients who recovered $(\mathrm{n}=25 ; \mathrm{AIN}=8$ and $\mathrm{CIN}=17$ ), the duration of neutropenia was 19.6 months (range 7-37) for AIN and 15.4 months (range 8-46) for CIN patients.

In terms of clinical course, only 13 patients with CIN and AIN (23.6\%) experienced infectious episodes during follow-up (Fig. 5). The most common site of infection was upper respiratory tract $(\mathrm{n}=11,39.3 \%)$. Other infections included lower respiratory tract $(n=4,14.3 \%)$, cutaneous abscess

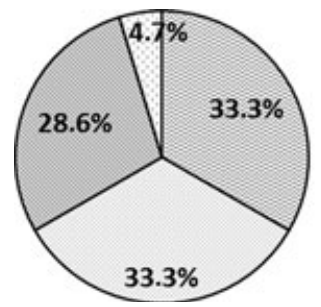

$\square$ Post-infective neutropenia $\square$ Chronic autoimmune neutropenia $\square$ Chronic idiopatic neutropenia $\square$ Severe congenital neutropenia

Figure 2 Etiologies of neutropenia in patients with ANC $<0.5 \times 10^{9} / 1$ at first evaluation $(n=42)$.

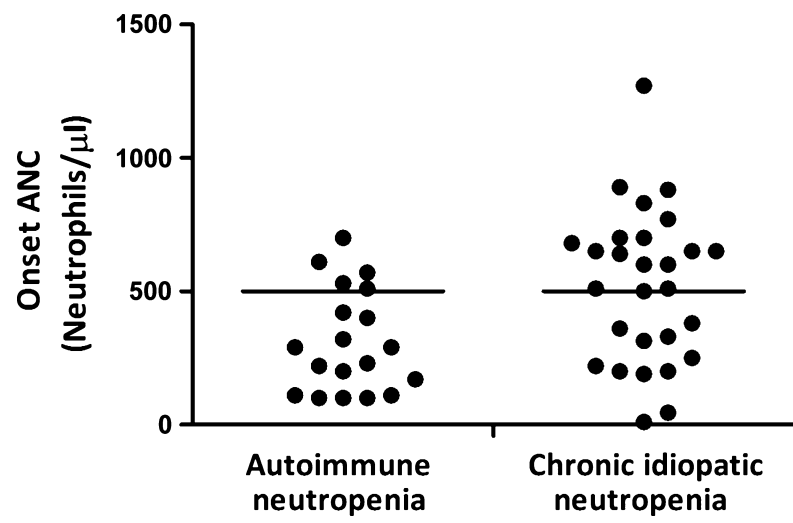

Figure 3 Onset ANC of patients with AIN and CIN: above $0.5 \times 10^{9} \%$ I, respectively, in the $26.3 \%$ and $60.7 \%$ of patients. Data were not available from 3 patients with AIN and 5 patients with CIN.

Table 3 Characteristics of congenital neutropenia

\begin{tabular}{|c|c|c|c|c|c|c|}
\hline Pt & Diagnosis & Gene mutation & $\begin{array}{l}\text { Age at onset } \\
\text { (months) }\end{array}$ & $\begin{array}{l}\text { ANC at } \\
\text { onset }(/ \mu l)\end{array}$ & $\begin{array}{l}\text { Mean ANC } \\
\text { during follow-up }(/ \mu l)\end{array}$ & $\begin{array}{l}\text { Treatment } \\
\text { with G-CSF }\end{array}$ \\
\hline 1 & SCN & ELANE & 1 & 690 & 642 & Yes \\
\hline 2 & SCN & ELANE & 9 & 320 & 440 & Yes \\
\hline 3 & SCN & ELANE & 1 & 720 & 1360 & No \\
\hline 4 & Hyper-IgM syndrome & CD40L & 42 & 680 & 1300 & No \\
\hline 6 & Suspected Cohen syndrome & $\begin{array}{l}\text { Molecular } \\
\text { analysis ongoing }\end{array}$ & 2.3 & 590 & 1580 & No \\
\hline
\end{tabular}

ND, not done. 
Table 4 Characteristics of autoimmune neutropenia (AIN) and chronic idiopathic neutropenia (CIN)

\begin{tabular}{lcc}
\hline & $\begin{array}{c}\text { Autoimmune } \\
\text { neutropenia (AIN) }\end{array}$ & $\begin{array}{c}\text { Chronic idiopathic } \\
\text { neutropenia (CIN) }\end{array}$ \\
\hline Total patients & 22 & 33 \\
Sex male/female & $8(36.4 \%) /$ & $15(45.5 \%) /$ \\
& $14(63.6 \%)$ & $18(54.5 \%)$ \\
Age at onset & 20 months (2-156) & 31 months \\
(mean, range) & & (range 1-141) \\
ANC at onset & $314 \mu(100-700)$ & $518 \mu l(10-1270)$ \\
(mean, range)* & & $814 \mu \mathrm{l}(45-3440)$ \\
ANC during & $712 \mu$ (80-2920) & \\
follow-up & & $10 \mu l$ \\
(mean, range) & & 20 months (8-73) \\
ANC nadir & $80 \mu l$ & $19(57.6 \%)$ \\
Follow-up period & 25 months (6-84) \\
(mean, range) & & \\
Patients with & $14(63.6 \%)$ & \\
follow-up beyond the & & \\
second year of age & & \\
\hline
\end{tabular}

$* p=0.01$.

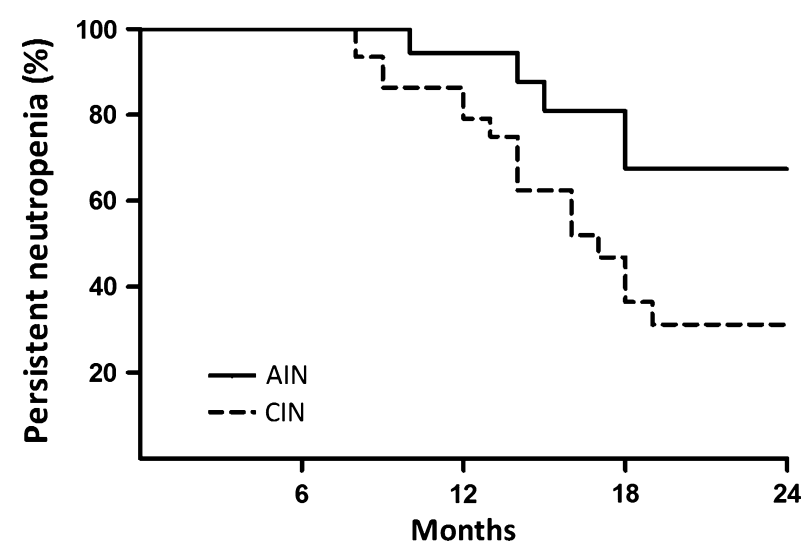

Figure 4 Remission rate of neutropenia in patients with AIN and CIN during a 24-month follow-up: AIN patients showed a lower remission rate $(p=0.027)$.

$(\mathrm{n}=3,10.7 \%)$, hordeolum $(\mathrm{n}=3,10.7 \%)$, stomatitis/oral ulcers $(\mathrm{n}=3,10.7 \%)$, and lymphadenitis $(\mathrm{n}=3,10.7 \%)$. One patient $(3.5 \%)$ presented with cellulitis. Severe infections requiring hospitalization occurred rarely. No patients with either AIN or CIN suffered from long-term consequences after infections. No significant difference was noticed in terms of mean ANC between patients with and without remission of neutropenia (respectively, $766 \mu \mathrm{l}$ and $778 \mu \mathrm{l}$ ), nor between patients with and without infections during follow-up (respectively, $721 \mu \mathrm{l}$ and $794 \mu \mathrm{l}$ ) (Fig. 6). Forty-five of 55 AIN/CIN patients were observed clinically without G-CSF treatment, while the remaining 10 patients were treated with G-CSF on the basis of their history of frequent or severe infections, with good clinical response (three of them received G-CSF only on demand). None of the patients was on antibiotic prophylaxis.

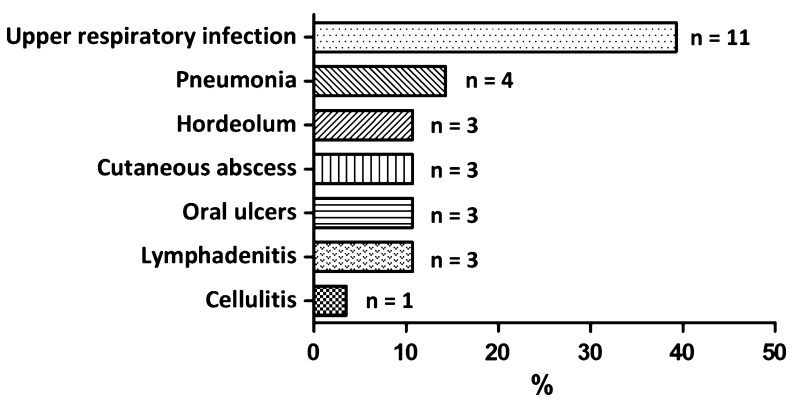

Figure $\mathbf{5}$ Location and frequency of infections in patients with AIN and CIN during follow-up ( $\mathrm{n}=13)$.

\section{Discussion}

The widespread use of routine biochemical assay has led to increased findings of neutropenia in pediatric practice. A special feature is that neutropenia is in fact a laboratory data, before representing a disease itself, and its origin and significance are extremely variable. Neutropenia may represent a predisposing factor to severe and life-threatening infections, as it is well known for patients with malignancies (15), but it can even be diagnosed incidentally in asymptomatic children, as complete blood count (CBC) is widely performed in outpatient settings.

Compared with the previous cohorts $(4,11,18,21)$, our populations includes a high proportion of patients with chronic neutropenia (63.5\%). Chronic neutropenia encompasses a wide spectrum of conditions, either as an isolated disorder or as a part of a syndrome (16). Patients with chronic idiopathic neutropenia and autoimmune neutropenia constitute the largest number of patients in this series, in addition to a number of less common forms such as severe congenital neutropenia, primary immunodeficiency, metabolic disorder, and inherited syndromes.

CIN and AIN have a similar clinical presentation and course. They both can be completely asymptomatic or lead to recurrent infections, often mild $(5,7)$. Only the detection of antineutrophil antibodies allows differential diagnosis, but they are not always searched in clinical practice, and furthermore, their detection presents technical difficulties $(19,20,23)$. Some authors hypothesized that part of CIN patients could be affected by a misrecognized AIN $(7,22,24)$. This hypothesis is supported by our experience: The systematic research of antineutrophil antibodies in 55 patients with isolated chronic neutropenia revealed 22 autoimmune forms $(40 \%)$. Such a prevalence is more frequent than previously reported $(5,7,12)$. Nevertheless, in the present study, the two groups showed subtle different features: Children with AIN usually presented with a lower ANC at onset and showed a lower remission rate during a 24-month follow-up. About this latter data, previous studies reported that AIN patients often spontaneously recover at the age of $2-3$ yrs $(9,19$, 20 ). As in the present study, only the $63.6 \%$ of patients with AIN were followed up beyond $2 \mathrm{yrs}$ of age, it is possible that a longer follow-up could have shown a higher remission rate in this patients group. Prolonged treatment with G-CSF was administered only to 7 patients with CIN or AIN, and all responded with elevated neutrophil count. A substantial number of our CIN/ AIN patients did not fulfill the strict criteria for the enrollment in 
(a)

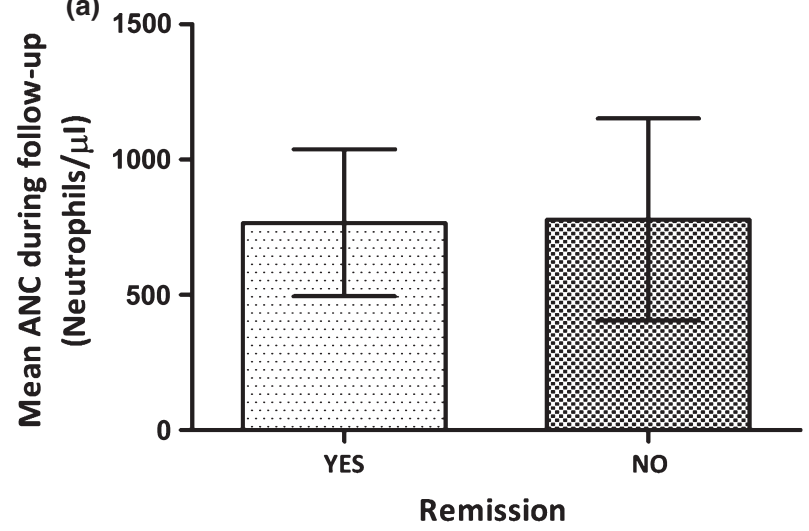

(b)

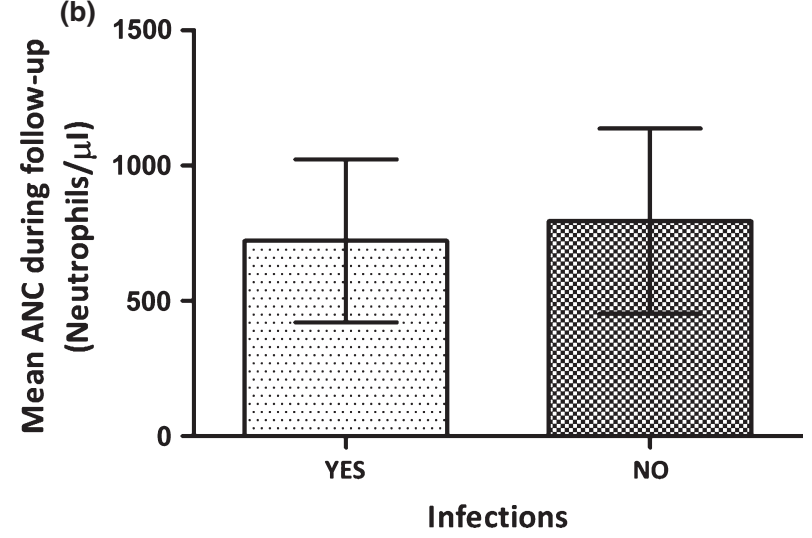

Figure 6 Mean ANC of patients with AIN and CIN during follow-up: No significant difference in ANC values was noticed between patients with and without remission of neutropenia (a), nor between patients with and without infections (b) during the study period. Bars indicate s.d.

the International Registry of Severe Chronic Neutropenia (SCNIR), showing ANC at onset $>0.5 \times 10^{9} / 1(60.7 \%$ of CIN and $26.3 \%$ of AIN) and a benign clinical course with few recurrent infections and frequent spontaneous remission.

In an effort to evaluate the clinical significance of neutropenia, we further analyzed the relations between $\mathrm{ANC}$ values and etiology, duration of neutropenia or infectious episodes during follow-up.

Severe neutropenia with $\mathrm{ANC}<0.5 \times 10^{9} / 1$ is a reason of concern and may be suspicious for congenital forms. However, in our experience, 4 of 6 patients with congenital neutropenia (including two with ELANE mutation) had ANC above $0.5 \times 10^{9} / 1$ on initial evaluations. On the other hand, onethird of patients with neutrophil level below $0.5 \times 10^{9} / 1$ at onset were diagnosed with a post-infectious acute form.

In the context of CIN and AIN, we further analyzed the mean ANC of patients in relation to infectious episodes and remission of the defect during follow-up. Patients with infections had mean ANC values comparable to asymptomatic patients, as well as patients who presented long-term remission had mean ANC values comparable to those with persistent neutropenia. Although the mean ANC does not take into account possible fluctuations of neutrophil number, which could partially affect these results, we could not demonstrate a direct and strong correlation between ANC and infections or $\mathrm{ANC}$ and long-term remission.

Acute neutropenia occurred in approximately one-third of our patient. An infection was the main reason for a transient defect, mainly by viral agents. Neutropenia in this group was frequently severe, in contrast to what previously reported (6). It is worth to point out that an infection can represent both the cause (post-infectious neutropenia) and the consequence (infection in a neutropenic patient) of neutropenia. Distinguishing these conditions could not be simple at first observation, but little anamnestic information (previous $\mathrm{CBC}$ and history of infections), some microbiological analysis, and observation of patient's ANC in the subsequent few months may help to define the diagnosis.

In the present study, drug-related neutropenia was diagnosed in only three children. However, the role of drug toxicity is very difficult to define, because the spectrum of potentially involved drugs is wide and it may also include unconventional drugs.

Regarding inherited neutropenia, there are currently several known causes that can be diagnosed at molecular level. In some cases, neutropenia is isolated (i.e., in patients with ELANE mutations), but in most of them, neutropenia is a part of a broader spectrum of signs and symptoms often involving extra-hematopoietic manifestations. Therefore, an accurate physical examination, including multidisciplinary evaluation, together with advanced laboratory tests and bone marrow analysis helps physicians to exclude or identify the majority of the known genetic forms of neutropenia. As suggested by the consensus guidelines on diagnosis from the Neutropenia Committee of the Marrow Failure Syndrome Group of the AIEOP (16), considering that chronic idiopathic neutropenia is an disorder of granulopoiesis characterized by prolonged neutropenia in the absence of any apparent underlying etiology, CIN patients should be placed on a monitoring program, and a diagnostic revaluation should be performed in case new elements suggestive of other diagnosis arise. In the future, the differential diagnosis of chronic idiopathic neutropenia will include a broad spectrum of genes, including recently identified genes. New approaches based on next-generation sequencing will eventually be adopted on a wider basis to facilitate the diagnosis of these patients. This will require an extensive validation of these new techniques and careful evaluation of the cost-benefit of this approach.

Considering the results of our study, it is worth to point out several limitations. Because of the high specialization of our Unit of Immunology and Infectious Disease and Unit of Hematology, it is likely that the sample study is not representative of the general pediatric population. The study was partially retrospective and little information may not be complete, depending on the compilation of medical records. Furthermore, we had to exclude patients with incomplete follow-up, who were likely affected by acute neutropenia (some did not return to visit after finding a normal ANC in outdoor exams). Our data were derived from a single tertiary care setting and cannot be extrapolated to an outpatient setting; thus, they reflect significant ascertainment bias inherent in any 
single institution study. These factors are likely responsible for the prevalence of chronic neutropenia in our cohort, in contrast to the previous series $(4,11,18,21)$. Another limitation comes from the age distribution of our cohort, with a high prevalence of infants $(n=50,48 \%)$. Before the age of $1 \mathrm{yr}$, neutropenia is defined with $\mathrm{ANC}<1 \times 10^{9} / 1$, while the severity classification distinguishes between mild, moderate, and severe neutropenia according to ANC values starting from $1.5 \times 10^{9} / 1$ (1). Therefore, neutropenic infants were only classified as moderate or severe defects. This could explain the higher prevalence of severe neutropenia in our cohort. Further studies focusing on neutropenia in infants are needed to identify a suitable classification for this age group.

In conclusion, our study confirms the great heterogeneity of diseases underlying neutropenia in children, although the frequency of the different etiologies observed in our patients could not reflect the real distribution of the causes in the general pediatric population. In our experience, we could not demonstrate a correlation between ANC level at onset and the nature of the underlying disorder. Similarly, ANC did not appear useful in predicting susceptibility to infections or longterm remission in patients with chronic acquired neutropenia. Neutropenia remains a disease of concern to pediatricians, requiring several laboratory investigations, prolonged followup, and in few cases, advanced molecular methods. Future prospective and structured studies are required to better assess the prevalence of different forms of neutropenia in general pediatric population.

\section{Acknowledgments}

We are grateful to Dr T. Lanza, G. Gaslini Children's Hospital of Genova, who performed the research of antineutrophil antibodies in some patients, and to Dr G. Savoldi, University of Brescia, who performed molecular analysis in some patients.

\section{Funding}

Supported in part by grants from Ministero della Salute (Giovani Ricercatori to AF), Fondazione Roma (to AI), and the European Commission (CELL PID, HEALTH-F5-2010261387 to AA and PR).

\section{Conflict of interest}

The authors have no conflict of interest to declare.

\section{References}

1. Segel GB, Halterman JS. Neutropenia in pediatric practice. Pediatr Rev 2008: 29: $12-23$.

2. Srdjan D, Saad S. Prevalence, phenotype and inheritance of benign neutropenia in Arabs. BMC Blood Disord 2009: 9: 3.

3. Haddy TB, Rana SR, Castro O. Benign ethnic neutropenia: what is a normal absolute neutrophil count? J Lab Clin Med 1999: 133: 15-22.

4. Alexandropoulou O, Kossiva L, Haliotis F, et al. Transient neutropenia in children with febrile illness and associated infectious agents: 2 years' follow-up. Eur J Pediatr 2013: 172: 811-9.

5. Wan C, Yu HH, Lu MY, et al. Clinical manifestations and outcomes of pediatric chronic neutropenia. J Formos Med Assoc 2012: 111: 220-7.

6. Karavanaki K, Polychronopoulou S, Giannaki M, et al. Transient and chronic neutropenias detected in children with different viral and bacterial infections. Acta Paediatr 2006: 95: 565-72.

7. Chung BHY, Chan GCF, Lee TL, et al. Chronic benign neutropenia among Chinese children. Hong Kong Med J 2004: 10: 231-6.

8. Bernini JC. Diagnosis and management of chronic neutropenia during childhood. Pediatr Clin North Am 1996: 43: 773-92.

9. Capsoni F, Sarzi Puttini P, Zanella A. Primary and secondary autoimmune neutropenia. Arthritis Res Ther 2005: 7: 208-14.
10. Perez-Mendez C, Molinos-Norniella C, Moran-Poladura M, Fernandez-Rodríguez E, Suarez-Castanon C, Solís-Sanchez G. Low risk of bacteremia in otherwise healthy children presenting with fever and severe neutropenia. Pediatr Infect Dis J 2010: 29: 671-2.

11. Vlacha V, Feketea G. The clinical significance of non-malignant neutropenia in hospitalized children. Ann Hematol 2007: 86: 865-70.

12. Yilmaz D, Ritchey AK. Severe neutropenia in children: a single institutional experience. J Pediatr Hematol Oncol 2007: 29: 513-8.

13. Alario AJ. Management of the febrile, otherwise healthy child with neutropenia. Pediatr Infect Dis J 1994: 13: 169-70.

14. Bonadio WA, Smith DS, Mathews S, Rock A. Clinical significance of newly documented neutropenia in febrile young infants evaluated for sepsis. Pediatr Infect Dis J 1991: 10: 407-8.

15. Pizzo PA. Management of fever in patients with cancer and treatment-induced neutropenia. $N$ Engl J Med 1993: 328: 1323-32.

16. Fioredda F, Calvillo M, Bonanomi S, et al. Congenital and acquired neutropenia consensus guidelines on diagnosis from the Neutropenia Committee of the Marrow Failure Syndrome Group of the AIEOP (Associazione Italiana Emato-Oncologia Pediatrica). Pediatr Blood Cancer 2011: 57: 10-7.
17. Dale DC, Bolyard AA, Schwinzer BG, et al. The severe chronic neutropenia international registry: 10-Year follow-up report. Support Cancer Ther 2006: 3: 223-34.

18. Husain EH, Mullah-Ali A, Al-Sharidah S, Azab AF, Adekile A. Infectious etiologies of transient neutropenia in previously healthy children. Pediatr Infect Dis J 2012: 31: 575-7.

19. Audrain M, Martin J, Fromont P, Prié N, Thomas C, Muller EJ. Autoimmune neutropenia in children: analysis of 116 cases. Pediatr Allergy Immunol 2011: 22: 494-6.

20. Sella R, Flomenblit L, Goldstein I, Kaplinsky C. Detection of anti-neutrophil antibodies in autoimmune neutropenia of infancy: a multicenter study. Isr Med Assoc J 2010: 12: 91-6.

21. Sheen JM, Kuo HC, Yu HR, Huang EY, Wu CC, Yang KD. Prolonged acquired neutropenia in children. Pediatr Blood Cancer 2009: 53: 1284-8.

22. Taniuchi S, Masuda M, Hasui M, Tsuji S, Takahashi H, Kobayashi Y. Differential diagnosis and clinical course of autoimmune neutropenia in infancy: comparison with congenital neutropenia. Acta Paediatr 2002: 91: 1179-82.

23. Bux J. Human neutrophil antigens. Vox Sang 2008: 94: 277-85.

24. Palmblad J, Papadaki HA. Chronic idiopathic neutropenias and severe congenital neutropenia. Curr Opin Hematol 2008: 15: 8-14. 